\title{
Ländliche Mittelzentren in Schleswig-Holstein - Kleine Städte vor großen Herausforderungen
}

\author{
Axel Priebs $^{1}$ \\ Online publiziert: 25 . Juli 2019 \\ (c) Der/die Autor(en) 2019
}

\section{Zusammenfassung}

Schleswig-Holstein ist ein Land mit einem hohen Anteil ländlicher Räume mit großer Entfernung zu den großen Städten. Deswegen kommt insbesondere außerhalb des Hamburger Umlandes den kleinen Städten eine tragende Rolle für die Sicherung gleichwertiger Lebensverhältnisse zu. Die von der Landesplanung festgelegten zentralen Orte haben den Auftrag, die flächendeckende Versorgung der Bevölkerung sicherzustellen. Normativ haben die Mittelzentren in der Landes- und Regionalentwicklung eine stabilisierende Bedeutung. Der Fokus des Beitrags liegt auf der Frage, vor welchen Herausforderungen diese Städte stehen, wobei insbesondere auf den städtischen Handel, das Verhältnis der Städte zu ihrem Umland und aktuelle Handlungserfordernisse eingegangen wird.

Schlüsselwörter Zentrale Orte $\cdot$ Gleichwertige Lebensverhältnisse $\cdot$ Regionalentwicklung $\cdot$ Kleinstädte .

Stadt-Umland-Kooperation

\begin{abstract}
Schleswig-Holstein is a state with a high proportion of rural areas with large distances to the big cities. Therefore, small towns play a key role in ensuring equivalent living conditions, especially outside the Hamburg environs. The central places defined by the state planning authority have the task of ensuring a comprehensive supply to the population. Normatively, the medium-sized centers have a stabilizing importance in the state and regional development. The focus of this article is on the challenges these towns face, focusing in particular on urban commerce, the relationship of towns to their environs and current needs for action.
\end{abstract}

Keywords Central places $\cdot$ Equivalent living conditions $\cdot$ Regional development $\cdot$ Small towns $\cdot$ Urban-environs cooperation

\section{Ländliche Mittelzentren als Leistungsträger in der schleswig-holsteinischen Landesplanung}

Die Kleinstädte sind seit einigen Jahren in den Mittelpunkt der raumwissenschaftlichen Forschung gerückt. Auch in der politischen Diskussion erhalten sie wieder ein größeres Gewicht, was vor allem mit ihrer potenziellen Entlastungsfunktion für überlastete Großstadtregionen, mit den aktuellen Diskussionen um gleichwertige Lebensverhältnisse in Deutschland und der Wiederentdeckung des Themas „Hei-

Axel Priebs

axel.priebs@univie.ac.at

1 Institut für Geographie und Regionalforschung, Universität Wien, Universitätsstr. 7, 1010 Wien, Österreich mat" zu tun haben dürfte. ${ }^{1}$ Aus dem Blickwinkel der Landes- und Regionalentwicklung ist dieses Interesse an den Klein- und zum Teil auch den Mittelstädten zu begrüßen, weil ein großer Teil dieser Städte normativ im zentralörtlichen System durch die Landesplanung als Mittelzentren ausgewiesen sind. Damit ist im schleswig-holsteinischen Landesentwicklungsplan (LEP) der Auftrag verbunden ,regional für die Bevölkerung ihres Verflechtungsbereichs die Versorgung mit Gütern und Dienstleistungen des gehobenen Bedarfs sicher zustellen“; darüber hinaus sind sie ,regionale Wirtschafts- und Arbeitsmarktzentren mit einem breit gefächerten Angebot an Arbeits- und Ausbildungs-

\footnotetext{
1 Vgl. zum aktuellen Forschungs- und Diskussionsstand das Themenheft „Kleinstädte - Chancen, Dynamiken und Potenziale“ des Bundesinstituts für Bau-, Stadt- und Raumforschung, (Informationen zur Raumentwicklung, Heft 6/2018)
} 


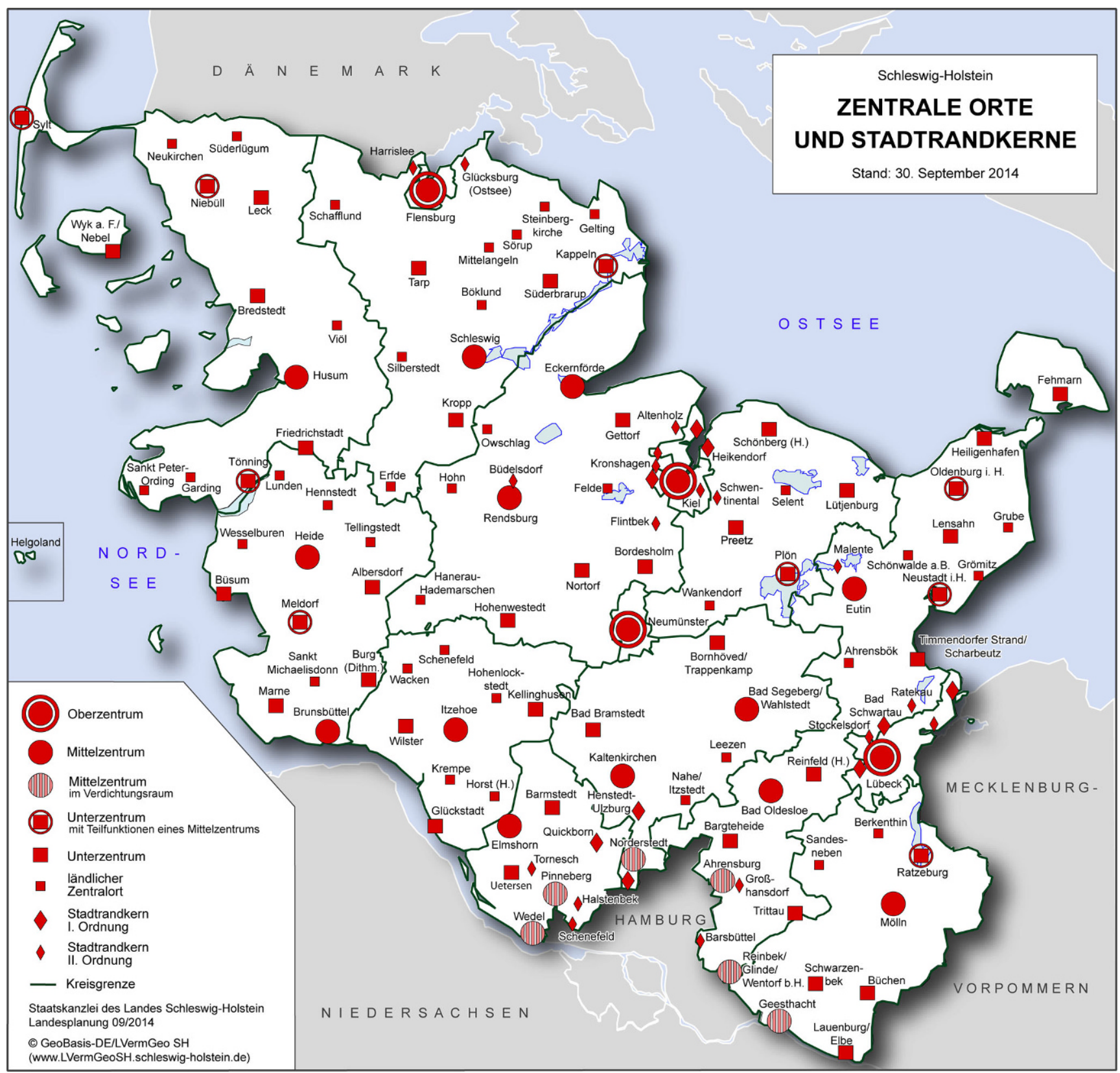

Abb. 1 Zentrale Orte und Stadtrandkerne Schleswig-Holstein, Stand: September 2014. (Karte: Staatskanzlei des Landes Schleswig-Holstein, Landesplanung)

plätzen“ (Innenministerium des Landes Schleswig-Holstein 2010, S. 38).

14 Städte sind in Schleswig-Holstein außerhalb des engeren Verdichtungsraums um Hamburg als Mittelzentren ausgewiesen, wobei die Einwohnerzahl zwischen 12.834 Einw. (Brunsbüttel) und 47.459 Einw. (Elmshorn) liegt. Weitere 9 Städte, die eigentlich als Unterzentren eingestuft sind, haben auch mittelzentrale Teilfunktionen und verdichten so das Netz der Städte mit mittelzentralen Funktionen (Abb. 1). Dass diese 23 Städte trotz gleichen Auftrags sehr unterschiedlich strukturiert sind und teilweise auch gegen den Bevölkerungsrückgang zu kämpfen haben, kann in diesem Kurzbeitrag nicht vertieft werden.

Bedauerlicherweise werden die aus dieser normativen Festlegung resultierenden Fragen, wie die Städte ihre zentralörtlichen Funktion ausfüllen und wie sie bei dieser Aufgabenerfüllung gestärkt werden können, in den aktuellen Klein- und Mittelstadtforschungen bislang nur wenig betrachtet. Dabei ist die Fragestellung in Schleswig-Holstein von besonderem Interesse, da den zentralen Orten seit über 50 Jahren eine tragende Rolle in der Landes- und Regionalentwicklung zugewiesen wird. Anders als die meisten Län- 
der unterstützt Schleswig-Holstein die Kommunen bei der Wahrnehmung übergemeindlicher Aufgaben mit Schlüsselzuweisungen aus dem kommunalen Finanzausgleich. AuBerdem wird die Leitfunktion des Handels in den zentralen Orten durch restriktive Regelungen zur Steuerung von Ansiedlungen großflächiger Einzelhandelseinrichtungen unterstützt, die „,wegen ihrer besonderen Bedeutung für die Zentralität nur in den zentralen Orten“ zulässig sind (Innenministerium des Landes Schleswig-Holstein 2010, S. 53). Unter- und Mittelzentren sollen Schwerpunkte der Infrastruktur und für Versorgungseinrichtungen sowie für die wohnbauliche und gewerbliche Entwicklung sein; das zentralörtliche System soll sicherstellen, „dass für alle Menschen im Land in zumutbarer Entfernung überörtliche Einrichtungen der Daseinsvorsorge erreichbar sind" (Innenministerium des Landes Schleswig-Holstein 2010, S. 35). Weil es sich aus Sicht der Landesregierung bewährt hat, müsse es ,im Hinblick auf die absehbaren demographischen Veränderungen" stabilisiert und gestärkt werden, um insbesondere in ländlichen Räumen ,langfristig die Daseinsvorsorge zu sichern" (Schleswig-Holsteinischer Landtag 2014, S. 4).

Dieser Beitrag will insbesondere mit Blick auf die Mittelzentren in ländlichen Regionen eine verstärkte wissenschaftliche und praktische Auseinandersetzung mit den Möglichkeiten zur Stabilisierung anregen. Denn normative Festlegungen sind zwar wichtig, für ihre Wirksamkeit müssen aber die Steuerungswirkungen bekannt sein und ggfs. Maßnahmen zu deren Unterstützung ergriffen werden. Hierbei wird nachfolgend sowohl auf die Instrumente des Landes als auf die Handlungsmöglichkeiten der öffentlichen und privaten Akteure vor Ort eingegangen, wobei auch die spezifischen schleswig-holsteinischen Rahmenbedingungen mit einer sehr kleinteiligen Gemeindestruktur zu beachten sind.

Die normative Strahlkraft der Mittelzentren resultiert aus der Bündelung vielfältiger Funktionen im Einzelhandel, in Bildung und Kultur, in der medizinischen Versorgung, in Verwaltung und Rechtsprechung sowie weiterer öffentlicher und privater Dienstleistungen und von Freizeit- und Sportangeboten. Entscheidend für den Erfolg eines Mittelzentrums im Einzelhandel ist, dass es entsprechend seinem überörtlichen Versorgungsauftrag neben der Bindung der eigenen Kaufkraft auch einen Kaufkraftzufluss von auBen generieren kann, was sich durch eine Zentralitätsziffer über 100 ausdrückt. Tatsächlich können in Schleswig-Holstein die „Mittelzentren außerhalb des Verdichtungsraums mit mehr als 10.000 Einwohnern einen teils erheblichen Kaufkraftzufluss verbuchen" (Industrie- und Handelskammer (IHK) Schleswig-Holstein 2017). Entsprechend weisen die ländlichen Mittelzentren Heide $(231,88)$, Rendsburg $(225,37)$, Husum $(222,80)$, Schleswig $(196,13)$, Itzehoe $(184,76)$, Eutin $(166,67)$ und Eckernförde $(163,97)$ sowie das Unterzentrum mit mittelzentralen Teilfunktionen

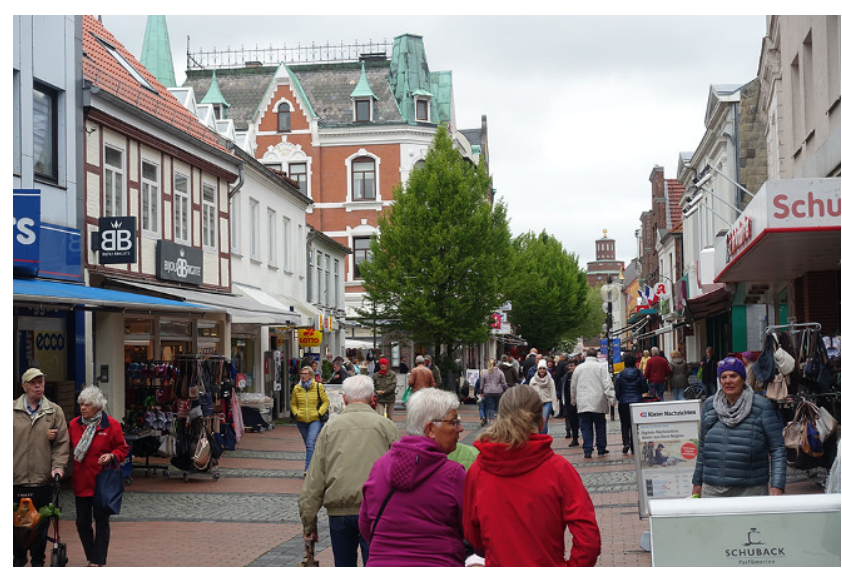

Abb. 2 Blick in die Eckernförder Fußgängerzone (Bild: Axel Priebs, Mai 2019)

Neustadt in Holstein $(179,49)$ beachtliche Zentralitätsziffern auf. Allerdings werden diese hohen Werte in einigen dieser Städte auch durch die touristische Nachfrage beeinflusst (Abb. 2).

Eine Vergleichbarkeit mit Städten anderer Bundesländer ist wegen des erwähnten engen Gebietszuschnitts der schleswig-holsteinischen Städte nur begrenzt möglich. Wegen der kleinteiligen Gemeindestruktur kommen die Städte mit der Funktion eines Mittelzentrums beispielsweise nicht an die Größenordnungen in Niedersachsen heran, wo es immerhin 16 derartige Städte mit über 30.000 Einw. in ländlichen Räumen gibt ${ }^{2}$. Während etwa die flächenmäBig besonders begrenzte Stadt Rendsburg aktuell auf knapp 30.000 Einwohner/innen kommt, leben im eng kooperierenden „Lebens- und Wirtschaftsraum Rendsburg“ mit seinen 13 Kommunen immerhin rund 70.000 Menschen. Die Enge der kommunalen Grenzen bewirkt nicht nur einen eingeschränkten Handlungsspielraum der Kernstadt und ein deutlich geringeres wirtschaftliches und politisches Gewicht, sondern erfordert auch eine ständige Auseinandersetzung mit den zahlreichen kleinen und kleinsten Gemeinden in der Nachbarschaft, worauf unten näher eingegangen wird.

\section{Herausforderungen für den Einzelhandel in den Mittelzentren}

Die genannten hohen Zentralitätsziffern der schleswig-holsteinischen Mittelzentren dürfen nicht darüber hinwegtäuschen, dass der stationäre Handel, der als Frequenzbringer eine elementare Funktion für die Innenstädte hat, vor allem durch den Onlinehandel vor großen Herausforderungen steht. Die IHK Kiel warnt davor, dass ,die stabilisierende Rolle dieses Stützpfeilers langsam wegzubrechen“

\footnotetext{
2 Niedersächsisches Institut für Wirtschaftsforschung: Regionalmonitoring Niedersachsen, Regionalreport (2007, S. 78).
} 
droht (Faltermeier 2017, S. 10). Tatsächlich lassen viele Innenstädte eine Reihe von Erosionserscheinungen erkennen. Langjährige Kaufhausleerstände, etwa in Rendsburg und Schleswig, aber auch Leerstände in anderen Städten sowie vernachlässigte öffentliche Räume vermitteln teilweise ein wenig erfreuliches Bild.

Deswegen stehen die Städte als auch der Handel vor der Aufgabe, laufend an ihrem Auftritt zu arbeiten. Den Städten ist zu empfehlen, im Dialog mit privaten Akteuren strategische Einzelhandelskonzepte und Visionen zu entwickeln. Um dem Innenstadthandel Planungssicherheit zu bieten, müssen die Städte das Planungsrecht gegen die Entstehung oder Ausweitung autoorientierter Konkurrenzstandorte am Stadtrand einsetzen. Auch für die Pflege des öffentlichen Raums und die Aufwertung der Aufenthaltsqualität sind primär die Städte verantwortlich. Der Handel muss zu mehr Zusammenarbeit kommen, was neben einheitlichen Öffnungszeiten und gemeinsamen Aktionen vor allem die Internetpräsenz betrifft (Jabs 2017). Diese ist wichtig, damit sich Kunden schon zu Hause über Angebote informieren oder Bestellungen aufgeben können. Dadurch können die Vorteile des Internets mit den Stärken des stationären Handels verbunden werden, die vor allem in der persönlichen Beratung, der Möglichkeit zur Ansicht der Waren und der direkten Mitnahme liegen. Außerdem wird immer deutlicher, dass ein wesentlicher Grund für den Einkauf im stationären Handel die Möglichkeit sein dürfte, anderen Menschen zu begegnen und z. B. mit ihnen zu kommunizieren (Boedecker 2019, S. 29).

Wie der stationäre Handel offensiv mit den Herausforderungen des Internethandels umgehen kann, zeigen Buchhandlungen, die über ihren Bestellservice im Internet ein bestelltes Buch am Folgetag bereitstellen können. Da beim Buchkauf die persönliche Beratung geschätzt wird, haben stationäre Buchhandlungen einen wesentlichen Vorteil gegenüber reinen Internetanbietern. Für die Lebensqualität einer Stadt ganz besonders interessant ist jedoch, dass Buchhandlungen zunehmend die Rolle örtlicher Kulturträger übernehmen. Auch in schleswig-holsteinischen Städten gehören Lesungen und andere Aktivitäten der Buchhandlungen zum unverzichtbaren Kulturangebot. Beispielsweise organisiert die Buchhandlung am Gänsemarkt im Mittelzentrum Eckernförde gemeinsam mit der Stadtbibliothek jährlich 6 bis 7 Autorenlesungen oder Buchvorstellungen. Ähnliche Aktivitäten haben sich in anderen Städten entwickelt, so im Mittelzentrum Bad Oldesloe, wo die Buchhandlung Willfang im Jahr 2016 auf die Ausrichtung von 111 Kulturabenden zurückblicken konnte (LN Online 13.04.2016).

Das Mittelzentrum Husum demonstriert seit Jahrzehnten, wie in einer großstadtfernen Region mit niedriger Bevölkerungsdichte eine Kleinstadt mit ca. 22.000 Einw. eine hohe Attraktivität entwickeln kann. Insbesondere das

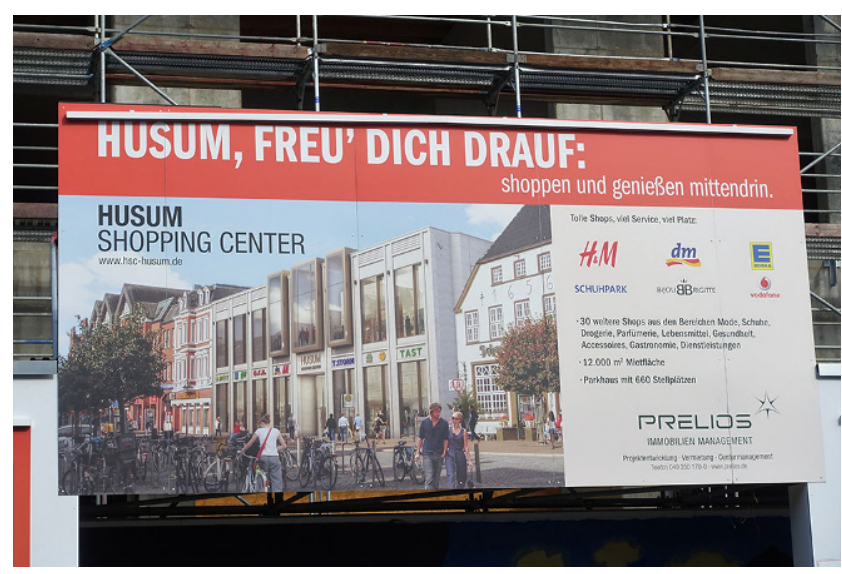

Abb. 3 Ankündigung für das künftige Shopping Center in Husum. (Bild: Axel Priebs, September 2018)

Textilkaufhaus C. J. Schmidt strahlt mit seiner Größe und seinem breiten Angebot weit in die ländliche Region aus. Zwar hat sich die Expansion dieses Betriebs durchaus auch auf Kosten kleinerer Anbieter vollzogen, doch verfügt die Innenstadt dadurch über einen starken Ankerbetrieb, wodurch beispielsweise auch die nördliche Ausdehnung des Innenstadtbereichs in die Neustadt mit ihren vielfältigen und kleinteiligen Geschäften möglich wurde. Auch der Zusammenbruch des Karstadt-Warenhauses konnte der Attraktivität der Innenstadt keinen Abbruch tun, weil der örtliche Handel hier mit einer Zwischennutzung eingestiegen ist, bevor mit der künftigen Einkaufspassage eine langfristige Folgenutzung auf den Weg gebracht wurde, die die Attraktivität Husums weiter erhöhen dürfte (Abb. 3).

Allerdings sind neue Malls und Passagen in der Innenstadt grundsätzlich differenziert zu beurteilen. Wo diese, wie in Husum, das klassische Angebot in geeigneter Lage und vertretbarer Größenordnung ergänzen, können sie positive Effekte auslösen, weil dadurch beispielsweise auch bei ungünstigem Wetter Flanieren und Aufenthalt in der Stadt möglich sind. Auch können sie örtlichen Betrieben Erweiterungsmöglichkeiten bieten und durch neue Anbieter für mehr Vielfalt sorgen. Um aber tatsächlich mit neuen Malls und Passagen Synergien und eine Stärkung der Innenstädte zu erreichen, ist bei derartigen Großprojekten eine ganz besondere planerische und städtebauliche Sensibilität erforderlich, was deren Standorte, Dimensionierung und Gestaltung betrifft.

\section{Das Stadt-Umland-Verhältnis als Herausforderung für die Klein- und Mittelstädte in Schleswig-Holstein}

Durch den engen Gebietszuschnitt ergeben sich für die Mittelzentren in Schleswig-Holstein spezifische Herausforde- 
rungen etwa bei der Entwicklung von Wohn- und Gewerbegebieten. Verteilungsprobleme zwischen den Kernstädten und ihren Randbereichen, die z.B. in Niedersachsen innerhalb des Stadtgebiets geklärt werden können, sind in Schleswig-Holstein meist interkommunale Fragen. Deswegen ist hier eine gute interkommunale Abstimmung eine zentrale Frage der Zukunftsfähigkeit der zentralen Orte und ihrer Leistungsfähigkeit auch für ihr Umland.

Dass das Verhältnis zwischen den Kleinstädten und ihren kommunalen Nachbarn nicht immer spannungsfrei ist, hat sich in Schleswig-Holstein bei der jüngsten Neuordnung der kommunalen Verwaltungen gezeigt. Die Landesregierung hatte 2002 einen Reformprozess angestoßen, weil sie erkannt hatte, dass die Struktur der Ämter, die für die kleineren Gemeinden die Verwaltung führen, nicht den Anforderungen an eine zeitgemäße Kommunalverwaltung entsprach. Das Ziel, Verwaltungseinheiten mit mindestens 8000 Einwohner/innen zu erreichen, führte wegen des hohen Maßes an Freiwilligkeit regional zu sehr unterschiedlichen Ergebnissen. Während etwa mit dem Amt Südtondern für die Stadt Niebüll und 29 weitere Gemeinden eine großräumige Verwaltungslösung für fast 40.000 Menschen erzielt wurde, erzielten andere Teilräume nur halbherzige Ergebnisse. Der Landesrechnungshof Schleswig-Holstein (2014) bewertet dabei besonders kritisch die Tendenz vieler Umlandgemeinden, Amtsverwaltungen um die Städte herum (,Kragenämter“) und damit Parallelstrukturen zu den Stadtverwaltungen auf- oder auszubauen. Als Mittelzentren sind von dieser problematischen Lösung beispielsweise die Städte Bad Segeberg, Eckernförde, Heide, Itzehoe und Ratzeburg betroffen. Laut Landesrechnungshof hat das Innenministerium diese Entwicklung zwar kritisch gesehen, aber nicht mit einer entsprechenden Regulierung untersagt (Landesrechnungshof Schleswig-Holstein 2014, S. 21); auch der Städteverband habe in dieser Struktur dezidiert eine Schwächung der Städte als zentrale Orte gesehen (Landesrechnungshof Schleswig-Holstein 2014, S. 22). Da sich Umlandgemeinden offenbar eigenständige Entscheidungsmöglichkeiten erhalten wollen, sind in Schleswig-Holstein Eingemeindungen wie die der (quasi insolventen) Gemeinde Schobüll in das Mittelzentrum Husum die Ausnahme geblieben. ${ }^{3}$ Als Sonderfall ist der Zusammenschluss der Stadt Burg auf Fehmarn mit den übrigen Inselgemeinden zu sehen, wodurch zum Jahresbeginn 2003 die neue, die gesamte Insel abdeckende Stadt Fehmarn (Unterzentrum mit 12.600 Einwohner/innen) als flächenmäßig zweitgrößte Gemeinde des Landes entstand.

Während angesichts des interkommunalen Abstimmungsbedarfs die Zurückhaltung des Innenministeriums in der Verwaltungsreform unverständlich ist, muss die Ausweisung von 14 Stadt- und Umlandbereichen als Ko- operationsräume um 10 ländliche Mittelzentren sowie 3 Unterzentren mit Teilfunktionen eines Mittelzentrums im Landesraumordnungsplan 1998 besonders positiv bewertet werden. Um die Entwicklung dieser Bereiche in geordnete Bahnen zu lenken, hat die Landesplanung dort die Erarbeitung von Gebietsentwicklungsplänen (GEP) initiiert und begleitet (vgl. Kühl und Liebrenz 1999; Innenministerium des Landes Schleswig-Holstein 2004). In diesem Prozess waren auch Vereinbarungen möglich, um bei begrenzten Entwicklungsmöglichkeiten der Kernstädte deren „Kontingente“ zur Siedlungsentwicklung im Umland zu verwenden. Allerdings ist der Kooperationsimpuls in den einzelnen Stadt-Umland-Bereichen sehr unterschiedlich weitergetragen worden. In Räumen, in denen entweder die Kernstädte vorrangig ein finanzielles Interesse an ihren Nachbargemeinden erkennen ließen oder diese nicht die Bedeutung der Städte für die Entwicklung des gemeinsamen Lebensraums anerkannten, sind die Kooperationsbestrebungen schnell im Sande verlaufen. Auch in anderen Räumen sind anfänglich vielversprechende Ansätze nicht weitergeführt worden. Umso bemerkenswerter ist es, dass aus dem damaligen Prozess zumindest 2 dauerhafte StadtUmland-Kooperationen entstanden sind, die im folgenden Kapitel kurz vorgestellt werden sollen.

\section{Erfolgreiche Stadt-Umland-Kooperationen in den Regionen der Mittelzentren Rendsburg und Heide}

Ausgelöst durch den Impuls der Landesplanung beschlossen 2003 im Wirtschaftsraum Rendsburg die Räte der Städte Rendsburg und Büdelsdorf sowie 11 weiterer benachbarter Gemeinden, eine gemeinsame Gebietsentwicklungsplanung (GEP) zu entwickeln, mit der eine bedarfsgerechte, geordnete und auf Schwerpunkte ausgerichtete Flächenentwicklung sichergestellt werden sollte (Abb. 4).

Der GEP-Prozess, der vom Bund als „Modellvorhaben der Raumordnung“ gefördert wurde, entwickelte sich $\mathrm{zu}$ einer intensiven interkommunalen Kooperation, die 2012 als „,Entwicklungsagentur für den Lebens- und Wirtschaftsraum Rendsburg“" in der Rechtsform der Anstalt des öffentlichen Rechts verfestigt wurde. Zu ihren Aufgabenschwerpunkten zählen neben dem Prozessmanagement das Marketing für den Wirtschaftsraum, die Flächen- und Verkehrsentwicklung, die Förderung der Einzelhandelsentwicklung, der An- und Verkauf von Flächen, die Akquisition von Fördermitteln sowie die Verwaltung eines von den Kommunen gespeisten Strukturfonds, aus dem Projekte mit regionaler Bedeutung und besonderen Nutzen für den Kooperationsraum finanziert werden; interessanterweise wurde da-

\footnotetext{
${ }^{3}$ Interview mit dem Bürgemeister der Stadt Husum am 14.02.2019.
} 


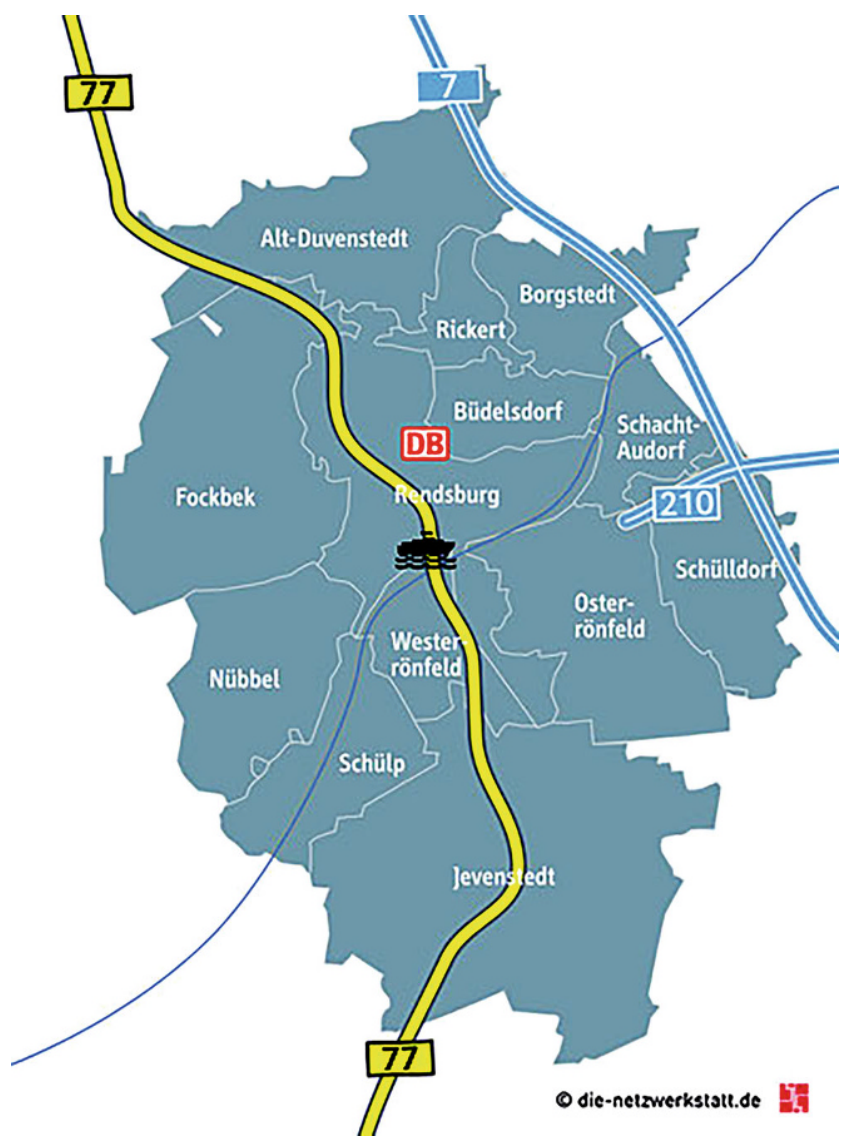

Abb. 4 Karte des Wirtschaftsraums Rendsburg. (Quelle: Entwicklungsagentur für den Lebens- und Wirtschaftsraum Rendsburg AöR)

raus die Sanierung eines Gymnasiums und des Theaters in Rendsburg finanziert. ${ }^{4}$

Auch in der Region Heide startete der Prozess mit der gemeinsamen Erarbeitung eines Gebietsentwicklungsplans. Daraus ergab sich jedoch nicht unmittelbar eine vertiefte praktische Kooperation, vielmehr wurde die Stadt Heide, wie erwähnt, zum Jahresbeginn 2008 durch ein neues Kragenamt „umzingelt“. Allerdings herrschte unter den Gemeinden eine erhebliche Unzufriedenheit mit der regionalen Wirtschaftsförderung, wodurch sie sich der regionalen Kooperation öffneten. Eine gemeinsame Klausur von Verantwortlichen aus Politik und Verwaltung in der Heider Region erbrachte 2009 den Durchbruch und initiierte einen zweijährigen, stark von der Landesplanung unterstützten Arbeitsprozess, an dessen Ende eine von allen 12 Kommunen unterzeichnete Kooperationsvereinbarung stand. 2013 wurde die Entwicklungsagentur Region Heide, ebenfalls in der Rechtsform der Anstalt des öffentlichen Rechts (AöR), gebildet, womit auch die Möglichkeit besteht, bei Bedarf auch hoheitliche Aufgaben zu übernehmen. Im Unterschied zu der nebenamtlich verwalteten AöR in Rendsburg hat die

\footnotetext{
${ }^{4}$ Interview mit der Geschäftsführung der AöR in Rendsburg am 15.02.2019. www.entwicklungsagentur-rendsburg.de
}

Heider Anstalt eine hauptamtliche Geschäftsführung sowie eigenes Personal. Eine zentrale Rolle spielt die Wirtschaftsförderung. Seit 2015 beschäftigen beide Agenturen gemeinsam einen „Fördermittelscout“.5

\section{Künftige Herausforderungen}

Künftig werden die Mittelzentren Schleswig-Holsteins, um die Herausforderungen in der Siedlungsentwicklung, der Wirtschaftsförderung und anderen öffentlichen Aufgabenbereichen zu bewältigen, noch stärker auf eine Kooperation mit dem Umland angewiesen sein. Um administrative Synergien zu schaffen und die mittelzentralen Funktionen der Städte zu stärken, sind unterschiedliche Lösungen möglich: intensivierte und organisatorisch verfestigte Kooperationen wie in den Regionen Heide und Rendsburg, großräumige Verwaltungsgemeinschaften wie im Raum Niebüll/ Südtondern oder Fusionen wie auf der Insel Fehmarn. Jede Stadt-Umland-Region sollte sich mit dieser Frage befassen und entscheiden, welche Lösung dem gemeinsamen Wirtschafts- und Lebensraum am meisten dient.

Für die Strahlkraft der Mittelzentren, aber auch der anderen zentralen Orte, haben die öffentlichen Einrichtungen eine wichtige Signal- und Stabilisierungsfunktion. Gerade deswegen ist für die Sicherung gleichwertiger Lebensverhältnisse in allen Teilräumen des Landes eine höhere Verbindlichkeit bei der Ausstattung mit diesen Einrichtungen erforderlich, wie sie von der Landesregierung angestrebt wird (Ministerpräsident des Landes Schleswig-Holstein 2016, S. 117). Verbindliche Mindeststandards für die zentralen Orte wären eine auch bundesweit interessante Innovation zur Stärkung des zentralörtlichen Systems und damit der Landes- und Regionalplanung. Wichtig ist auch, dass in der anstehenden Weiterentwicklung des kommunalen Finanzausgleichs in Schleswig-Holstein die gesonderte Dotierung der zentralen Orte bestehen bleibt; sinnvoll dürfte deren Verknüpfung mit den angestrebten Mindeststandards sein.

Funding Open access funding provided by University of Vienna.

Open Access Dieser Artikel wird unter der Creative Commons Namensnennung 4.0 International Lizenz (http://creativecommons.org/ licenses/by/4.0/deed.de) veröffentlicht, welche die Nutzung, Vervielfältigung, Bearbeitung, Verbreitung und Wiedergabe in jeglichem Medium und Format erlaubt, sofern Sie den/die ursprünglichen Autor(en) und die Quelle ordnungsgemäß nennen, einen Link zur Creative Commons Lizenz beifügen und angeben, ob Änderungen vorgenommen wurden.

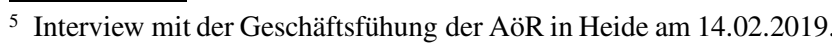
www.region-heide.de
} 


\section{Literatur}

Boedecker JM (2019) Ladensterben: Die Stadt neu erfinden. Kommunal 07/08:28-29

Faltermeier L (2017) Gemeinsam für lebendige Innenstädte. Wirtschaft zwischen Nord- und Ostsee, Bd. 12, S 10

Industrie- und Handelskammer Schleswig-Holstein (2017) Kennzahlen des Einzelhandels Schleswig-Holsteins: Kaufkraft, Umsatz und Zentralität 2017. Flensburg/Kiel/Lübeck

Innenministerium des Landes Schleswig-Holstein (Hrsg) (2004) StadtUmland-Konzepte in Schleswig-Holstein - Empfehlungen für die kommunale Praxis. Kiel

Innenministerium des Landes Schleswig-Holstein (Hrsg) (2010) Landesentwicklungsplan Schleswig-Holstein 2010. Landesplanung in Schleswig-Holstein, Bd. 33. Kiel
Jabs A (2017) Im Netz Kunden gewinnen. Wirtschaft zwischen Nordund Ostsee, Bd. 12, S 8

Kühl C, Liebrenz F (1999) Durchbruch bei der interkommunalen Zusammenarbeit in Schleswig-Holstein. Raumforsch Raumordn 57(2/3):201-202

Landesrechnungshof Schleswig-Holstein (2014) Ergebnis der Verwaltungsstrukturreform im kreisangehörigen Bereich. Kiel. www. landesrechnungshof-sh.de/file/verwstrukturreform2014.pdf

Ministerpräsident des Landes Schleswig-Holstein (2016) Landesentwicklungsstrategie Schleswig-Holstein 2030 (Entwurf). Kiel

Niedersächsisches Institut für Wirtschaftsforschung (2007) Regionalmonitoring Niedersachsen, Regionalreport

Schleswig-Holsteinischer Landtag (Hrsg) (2014) Raumordnungsbericht 2014. Bericht der Landesregierung - Ministerpräsident. 18. Wahlperiode, Drucksache 18/2082 